\title{
Intracerebral Implantation of Ionic Synthetic Hydrogels: Effect of Polar Substrata on Astrocytosis and Axons
}

\author{
S. Woerly ${ }^{1}$, C. Lavallée ${ }^{2}$ and R. Marchand ${ }^{1}$ \\ ${ }^{1}$ Centre de recherche en neurobiologie, Hôpital de l'Enfant Jesus et Université Laval, 1401 18e rue, Québec \\ (Québec), G1J $1 Z 4$ and ${ }^{2}$ Centre de recherche en Sciences et en Génie des macromolécules, \\ Université Laval, Québec (Québec), G1K 7P4, Canada
}

\begin{abstract}
SUMMARY
In previous studies, hyperporous synthetic hydrogels of poly(glyceryl methacrylate) or p(GMA), containing bioadhesive substrates of collagen, were implanted into rat cerebral tissue in order to provide systems of oriented guidance channels for directing the growth of the scar and axons $/ 28 /$. In the present study, ionic p(GMA)collagen hydrogels containing polar chemical groups, either basic amino groups or acidic carboxyl groups, were evaluated for their tolerance and their effects on the brain scarring response and axonal reactivity after long-term implantation in the cerebral cortex. In all animals, the implants were well tolerated. Although both types of gels influenced the astroglial reaction near the bioimplant, hydrogels carrying carboxyl groups had the strongest influence on the elongation, the direction and the organization of astrocytic processes so that a glial matrix could form in regions of the gel. Extracellular material (e.g. reticulin) was also deposited into the gels carrying carboxyl groups. Although cortical
\end{abstract}

Reprint address:

S. Woerly (present address)

Department of Biological Sciences

University of Keele

Keele ST5 5BG

Staffordshire, UK nerve fibers in the surrounding tissue showed a regenerative response, extending onto or into the matrices, this behavior seemed to depend more on the organization of the astrocytic scar imposed by the gel than on the type of gel. We conclude that matrices carrying negatively charged groups influence favorably the astrocytosis and the deposition of connective tissue, and that this approach represents a new avenue in attempting to modulate the brain scar formation.

\section{KEY WORDS}

poly(glyceryl methacrylate), ionogenic hydrogels, intracerebral implantation, scar tissue, axonal regeneration

\section{INTRODUCTION}

Hydrogels are three-dimensional cross-linked networks of hydrophilic polymers swollen in water or biological fluids. The biocompatibility of synthetic hydrogels was first established by Wichterle and Lim in 1960/32/ and a wide range of hydrophilic polymers have since received a great deal of interest for biomedical applications as tissue replacement and regeneration templates $/ 24 /$. 
Our early studies, dealing with the implantation of synthetic hydrogels of poly(glyceryl methacrylate) $[\mathrm{p}(\mathrm{GMA})]$ into the brain, have shown that such material provides a three-dimensional lattice network of guidance channels (matrix) that support and direct cell migration and axonal growth /34/. Indeed, adhesion and growth, which are basic biological mechanisms of morphogenesis and regeneration, depend upon the provision of a suitable substratum. Hence, a variety of synthetic guidance channels have been used experimentally to promote nerve regeneration in the peripheral nervous system /19,21/ and in the central nervous system $/ 16 /$. We have previously reported that tissue ingrowth and scar deposition were directed inside the polymer network for $\mathrm{p}(\mathrm{GMA})$ matrices having $60 \%$ of fractional porosity and an average pore size of 13 $\mu \mathrm{m}$. The present study describes an investigation carried out with similar $\mathrm{p}(\mathrm{GMA})$ hydrogels but containing polar groups. Either dimethylaminoethyl methacrylate (DMAEMA) as a carrier of basic amino groups or methacrylic acid (MAA) as a carrier of acidic carboxyl residues was introduced into $\mathrm{p}(\mathrm{GMA})$ hydrogels. In contact with the polar media, these materials acquire electrical charges by ionization of amino and carboxyl groups: $p($ GMA)-DMAEMA becomes positively charged and $p(G M A)-M A A$ negatively charged. Since cells, including neurons, respond actively to an applied electrical field (galvanotaxis) $/ 6 /$, it would be expected that the neural tissue would be sensitive to implanted ionic hydrogels. However, because of the unwillingness of cells to attach to the surface of the synthetic polymers $/ 9 /$, and as reported in our previous study $/ 34 /$, collagen was introduced into the hydrogels in order to provide bioadhesive properties to the polymer surfaces. The objectives of this work were then to establish the tolerance of ionic $p(G M A)$ implants and the effect of functional groups on the reactivity of the cell constituents of the neural tissue after long-term survival periods. The results of these experiments are discussed in the context of surface polarity and galvanotaxis on cell movement and axonal growth. The present study is part of a larger work, the aim of which is to develop guidance channels containing polymer matrices that could modulate the brain scarring response and promote axonal growth. Some of the present results have already been published in abstract form $/ 33 /$.

\section{MATERIAL AND METHODS}

\section{Preparation of hydrogels}

The following chemicals, glycidyl methacrylate, ethylene glycol dimethacrylate (EGDMA), DMAEMA, MAA, ammonium persulfate and sodium metabisulfite, were purchased from Aldrich. Type I collagen was obtained from Collagen Corporation, Palo Alto, CA.

Pure GMA was obtained by hydrolysis of the glycidyl methacrylate with sulfuric acid according to the method described by Refojo and the reader is referred to this reference for details of the procedure $/ 25 /$. However, the GMA monomer was further purified by distillation under dynamic vacuum.

The $\mathrm{p}(\mathrm{GMA})$ hydrogels were prepared by solution polymerization containing the monomer, the cross-linking agent EGDMA and either DMAEMA or MAA (Fig. 1). Distilled water served as solvent. The concentrations of GMA and EGDMA were $30 \%$ and $1.23 \%$ (w/w) respectively corresponding to values that yield hyper- and macroporous matrices and for which we obtained the best biological properties in our previous study. DMAEMA or MAA was added to the monomer mixture at a concentration of 10 and $15 \%$ ratio to the monomer respectively. At these concentrations, ionic groups are non-toxic and have been shown to influence the tissue reaction in non-neural tissues $/ 1,30 /$. The polymerization reaction was initiated at $60^{\circ} \mathrm{C}$ with ammonium persulfate ( $6 \%$ solution) and sodium metabisulfite ( $12 \%$ solution) in a 0.37 weightpercentage ratio to the monomer.

The hydrogels were washed in distilled water for four weeks and sterilized by boiling in water. Collagen was introduced into the polymers by physical adsorption in order to provide adhesive surface properties to the matrices. This was performed by allowing the dehydrated hydrogels to swell in a sterile neutral collagen solution for a minimum of four hours at $4^{\circ} \mathrm{C}$. The collagen solution was prepared by mixing 8 vol. of acid-sol- 
uble type I collagen with 1 vol. of $1.3 \mathrm{M} \mathrm{NaCl}$ in $0.2 \mathrm{M}$ monophosphate and $1 \mathrm{vol}$. of $0.1 \mathrm{M} \mathrm{NaOH}$.
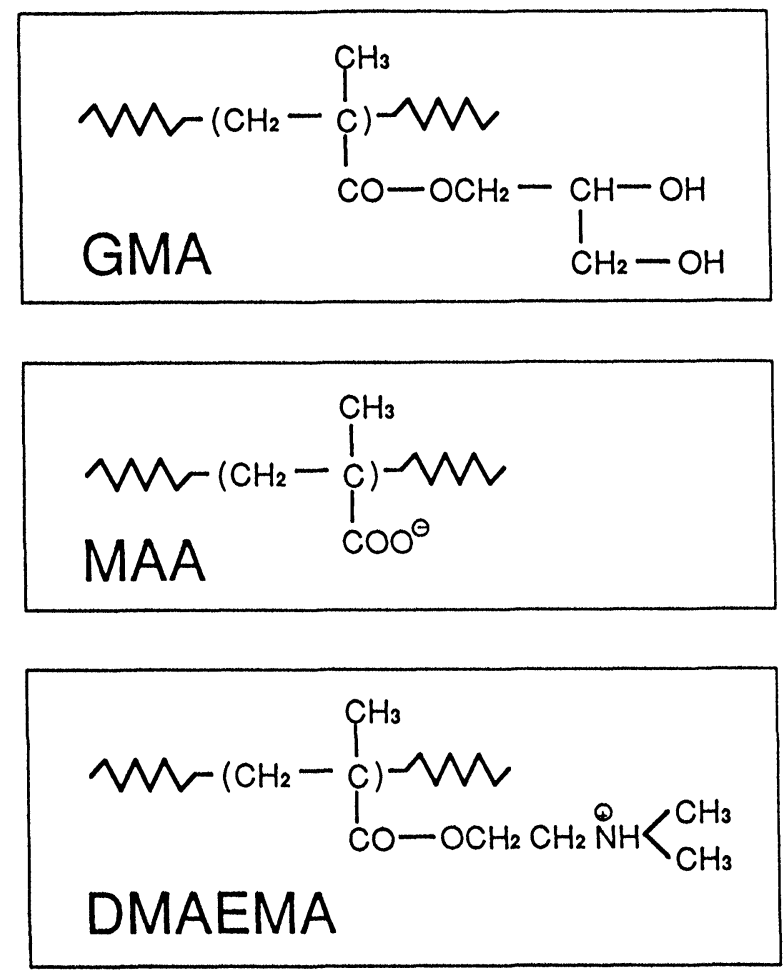

Fig. 1: Structure of the GMA monomer, and the MAA and DMAEMA comonomers. The charges of the carboxyl and amino groups refer to the ionization state of the groups at $\mathrm{pH} 7.4$

\section{Implantation procedure}

Eight young female Sprague-Dawley rats (250-300 g) were used as recipients. The animals were anesthetized with an intramuscular injection $(1 \mathrm{ml} / \mathrm{kg})$ of a solution containing ketamine hydrochloride $(87 \mathrm{mg} / \mathrm{ml})$ and xylazine (13 $\mathrm{mg} / \mathrm{ml}$ ) and placed in a stereotactic head frame. Using microsurgical techniques and through a midline scalp incision, a small craniotomy ( $3 \times 3$ $\mathrm{mm}$ ) was performed and the left parietal cortex exposed. The dura was incised and folded. A cross-shaped incision about 2-2.5 mm deep was performed in the cortex using a No. 11 surgical blade. After $\mathrm{h} \geqslant$ mostasis with thrombin-soaked gelfoams, hydrogel samples $(1 \times 1 \mathrm{~mm})$ were introduced with a jeweler's forceps through the cross-shaped incision of the cortex and the bone flap was immediately replaced and the scalp clipped. The types of hydrogels implanted are given in Table 1.

\section{Histology}

At 12 and 18 weeks post-implantation, the animals were transcardially perfused with a $0.9 \%$ $\mathrm{NaCl}$ solution followed by $4 \%$ paraformaldehyde- $0.5 \%$ glutaraldehyde in phosphate buffer (200 ml each). The brains were postfixed overnight, transferred in $30 \%$ buffered sucrose and coronally cut at $30 \mu \mathrm{m}$ with a freezing microtome. The histological techniques used were: phosphotungstic acid-hematoxylin (PTAH) for glial cells and Bodian's method for nerve fibers. Collagen was detected using the picrosirius-polarization method. Sirius red is an acidic dye which strongly reacts with collagen producing a marked increase of its intrinsic birefringence under polarized light $/ 12,13 /$.

\section{Glial fibrillary acidic protein (GFAP) immuno- cytochemistry}

Sections chosen for immunostaining were incubated, free-floating, in $10 \%$ normal goat serum in PBS for $30 \mathrm{~min}$. The sections were then washed in PBS for 10 minutes and incubated overnight and under agitation in the primary antiserum (diluted in PBS 1:40) (Sigma, St Louis). After three 10 minute washings in PBS, the sections were incubated in the secondary antiserum coupled to fluorescein isothiocyanate (FITC) (Sigma, St Louis) for $2 \mathrm{~h}$ under agitation. After three 10-minute rinses in PBS, the sections were mounted on chromalum coated slides, dried and coverslipped with the DPX mounting medium. The sections were examined with a fluorescence microscope.

\section{Scanning electron microscopy (SEM)}

Several non-implanted samples were lyophilized and coated with gold. They were analyzed with a Jeol T 300 scanning electron microscope at $15 \mathrm{KV}$. 
TABLE 1

Composition of ionic $\mathrm{p}(\mathrm{GMA})$ hydrogels and evaluation of the neural tissue response

\begin{tabular}{|c|c|c|c|c|c|c|}
\hline rat & copolymer & polar groups & $\begin{array}{l}\text { ionic } \\
\text { structure }\end{array}$ & $\begin{array}{l}\text { survival } \\
\text { periods }\end{array}$ & $\begin{array}{l}\text { astrocyte } \\
\text { projections }\end{array}$ & $\begin{array}{l}\text { axonal } \\
\text { reaction }\end{array}$ \\
\hline 1 & $15 \% \mathrm{MAA}$ & carboxyl & anionic & 12 weeks & + & I \\
\hline 2 & $15 \% \mathrm{MAA}$ & carboxyl & anionic & & ++ & I and $\S$ \\
\hline 3 & $10 \%$ DMAEMA & amino & cationic & & + & ก \\
\hline 4 & 10\% DMAEMA & amino & cationic & & + & $\pi$ and $\S$ \\
\hline 5 & $15 \% \mathrm{MAA}$ & carboxyl & anionic & 18 weeks & ++ & $\pi$ and $\S$ \\
\hline 6 & $15 \% \mathrm{MAA}$ & carboxyl & anionic & & +++ & I and $\S$ \\
\hline 7 & 10\% DMAEMA & amino & cationic & & + & ก \\
\hline 8 & $10 \%$ DMAEMA & amino & cationic & & ++ & $\pi$ \\
\hline
\end{tabular}

+ limited at the brain-implant interface

invasion of the gel implant: to the margin $(++)$, to the central region $(+++)$

I axonal sprouts at the interface

$\S$ axons projecting into the gel implant

\section{RESULTS}

\section{Morphology of the hydrogels}

Macroscopically, the swollen hydrogels were transparent materials with smooth surfaces. Under SEM, there were no noticeable differences between the hydrogels, both types showing a three-dimensional porous structure with intercommunicating channels (Fig. 2). The surface porosity of the gels was heterogeneous with a pore size above or equal to $10 \mu \mathrm{m}$.

\section{Findings at autopsy}

All animals survived to the scheduled date of sacrifice. Neither neurological deficits nor abnormal behaviors were observed in any of the rats. There was no necrotic zone observed around the implants at the time of brain retrieval. Macroscopically, the hydrogel implants seemed well integrated to the host tissue and there was no apparent degradation of their struc- ture (Fig. 2). This was confirmed using the light microscope.

\section{Histological findings}

\section{General}

The implants were entirely contained within the frontoparietal cortex and could occasionally reach the cingulum or penetrate the corpus callosum and the superficial hippocampus. The tolerance of samples carrying anionic or cationic groups was excellent and virtually the same for all hydrogel implants. There were no signs of pronounced inflammatory response nor of tissue toxicity. The macrophage reaction was weak as judged by the presence of only a few resident phagocytic cells around the implantation site at the time of retrieval. The histological analysis showed that there were no marked differences in the tissue reaction with respect to the survival periods. The host tissue reaction consisted of 


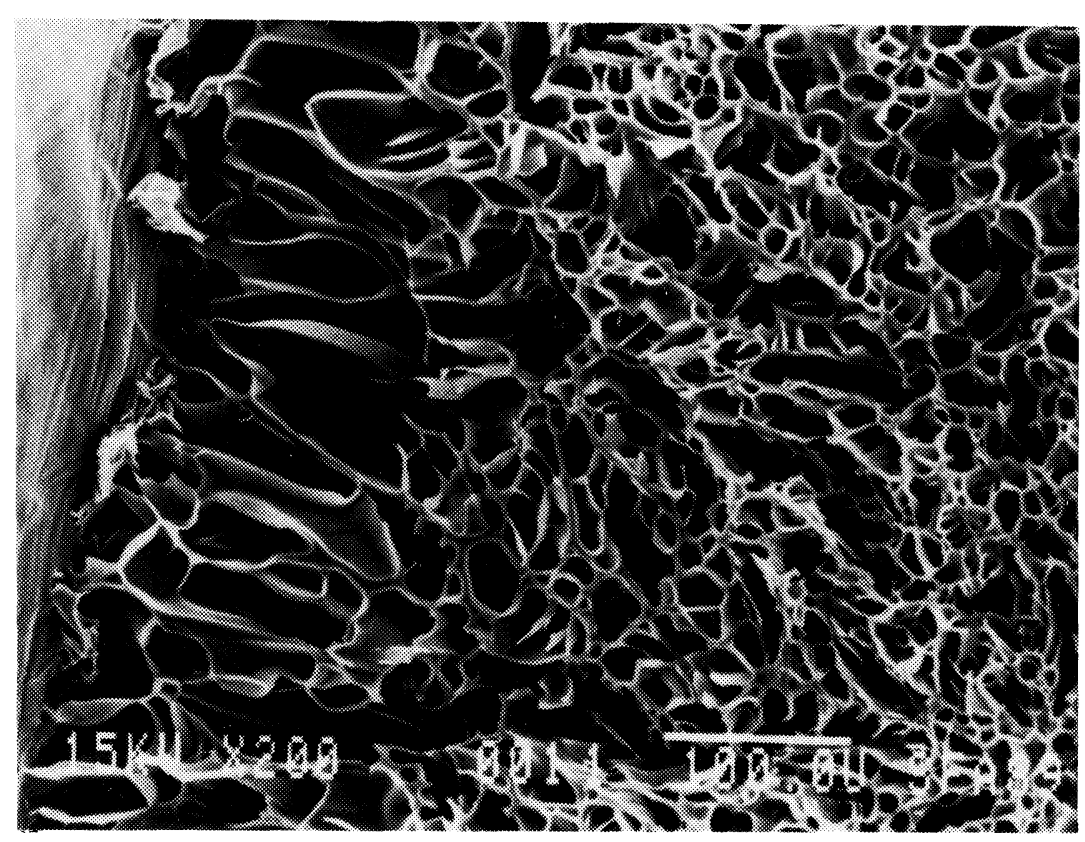

Fig. 2: Scanning electron micrograph of a p(GMA)-MAA hydrogel. The gel shows a highly porous structure with oriented and interconnected channels.

astrocytosis, connective tissue proliferation, mesenchymal cell migration, nerve fiber sprouts and capillary proliferation around, onto and/or into the implant. Encapsulation of the polymer implants occurred only when there was a partition between the marginal regions of the gel implant and the host tissue (e.g. Fig. 5A).

The brain-bioimplant interface was characterized by the apposition of the polymer matrix to the host tissue. At high magnification, the interface was constituted of glial cells and glial processes, capillaries and by the deposition of elastic (stained with PTAH) and reticular fibers, and collagen (vide infra). All these elements formed a zone of transition and were spatially organized to constitute a loose matrix bridging the surface of the polymer to the host neural tissue (Fig. 3A).

\section{The astrocytosis}

The glial reaction that developed around the hydrogels was markedly different from that associated with the stab wound which often reached the cingulum and/or the corpus callosum under the implantation site (Fig. 3C, area 3). At this later level, the glial reaction was constituted by a dense aggregation of fibrous astrocytes which tended to spread into the brain parenchyma (Fig. 3C). The astrocytic reaction was different around the hydrogel implants. The astrocytosis was moderate as judged by the low density of astroglial cells and by the narrow zone of reactivity, and there was no spreading of the astrocytosis with the longest survival period. This was true even in the cases where the implants were in contact with the cingulum and/or the corpus callosum. The glial reaction was characterized morphologically by the proliferation and the extension of numerous astrocytic processes in the immediate vicinity of the hydrogels. This was readily observed, regardless of the ionic composition of the hydrogels, either in PTAH stained (e.g. Fig. 5B) or GFAP immunolabeled brain sections (Fig. 3B). In addition, there was a striking polarization of the processes of the reactive astrocytes with respect to the surface of the hydrogels, but this could be observed only in histological brain sections passing near to the brainimplant interface. Figure $5 \mathrm{~B}$ gives an example of such a hydrogel where the astrocytic processes are arranged perpendicularly with respect to one 

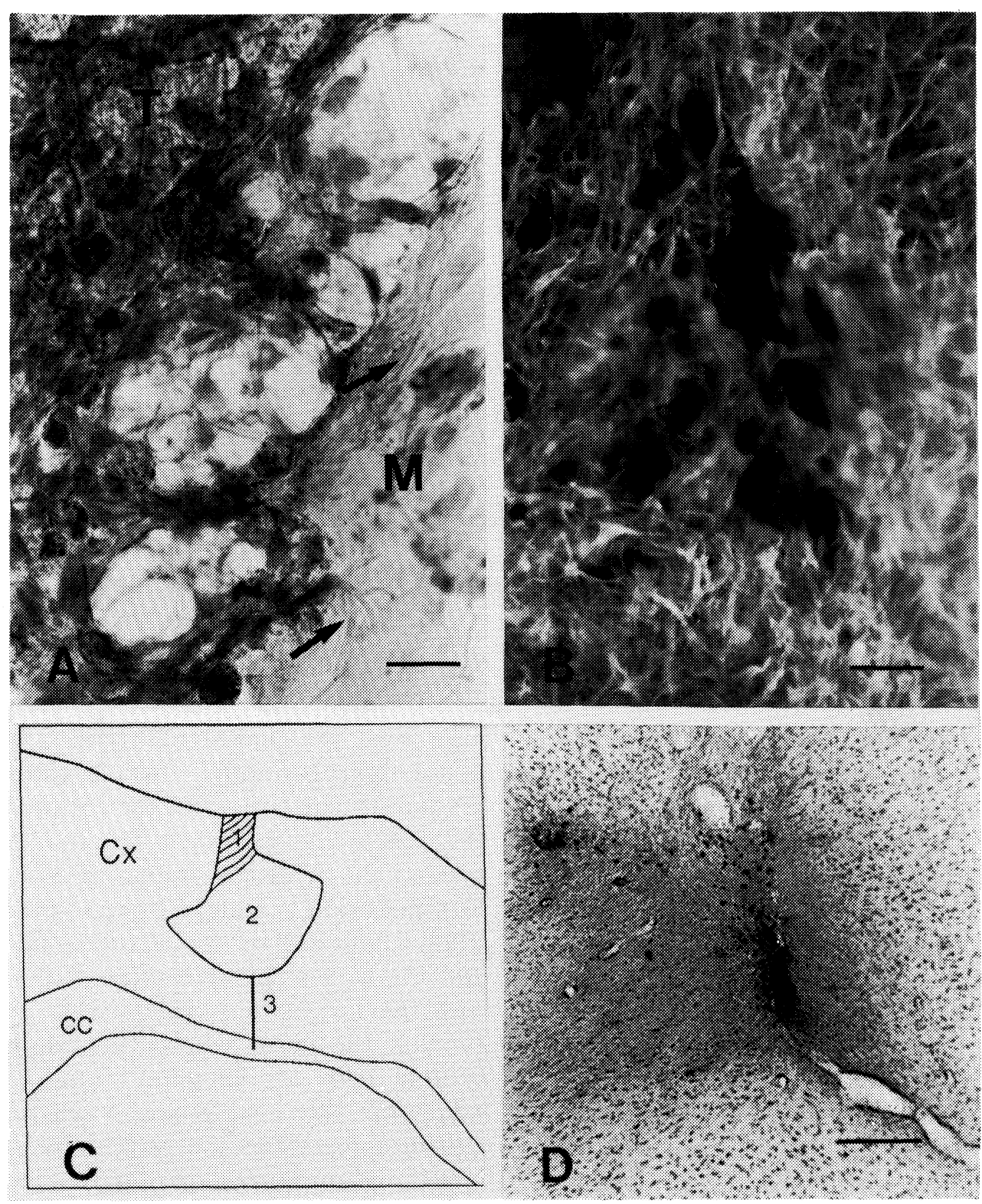

Fig. 3: $\quad A(P T A H)$ and $B$ (GFAP immunostaining) correspond to $p(G M A)-M A A$ gels implanted 12 weeks before. A, high magnification of the brain bioimplant interface showing zone of contact between the tissue (T) - mostly astrocytic processes - and the surface of the gel (M). Astrocytic processes (arrows) can be seen to penetrate the matrix $(M)$. In $B$, reactive astrocytes surround the implanted gel and send processes onto and into the implant. The fuzzy zones of the microphotograph correspond to the different refractive index of the gel with respect to the tissue. $C$ is a schematic representation of the general organization of the implantation site. 1 - mesenchymal tissue closes the opening of the implantation cavity; 2 corresponds to the implanted gel and 3 is the prolongation of the blade cut. cc, corpus callosum; CX, cortex. D (PTAH) illustrates the strong glial reaction at level 3 of figure C. Scale bars: A, $10 \mu \mathrm{m} ; \mathrm{B}, 40 \mu \mathrm{m}$ and D, $100 \mu \mathrm{m}$. 
surface of the hydrogel. In addition, the astrocytic processes were seen invading the gel implants and this was observed mainly at 18 weeks postimplantation (Table 1). However, there were marked differences between the two types of implants regarding the extent to which the astrocytic processes penetrated the gel as well as regarding their organization. Although reactive astrocytes projected numerous processes towards the marginal zone of the gels, it was only with $\mathrm{p}(\mathrm{GMA})-\mathrm{MAA}$ gels that there was evidence that the extent and the organization of the astrocytic projections were influenced by the polymer implant. The processes invaded the deep structures of the gel so that they had even constituted a glial matrix inside domains of a $\mathrm{p}(\mathrm{GMA})-\mathrm{MAA}$ hydrogel after 18 weeks (Fig. 4A, Table 1). Moreover, the processes seemed to follow the structure of the gel and, at high magnification, individual astrocytic processes were seen coursing into the gel, sometimes in association with blood vessels (Fig. 4B). For the p(GMA)DMAEMA hydrogels, the astrocytic processes extended only to the interface, onto the surface of the gel and into marginal regions, even after 18 weeks postimplantation.

\section{Deposition of extracellular materials}

Under polarized light, the brightness of the birefringence as well as the various polarization colors which circumscribed the matrices were suggestive of the deposition and of the organization of newly formed collagen at the brainbioimplant interface, since the collagen stock that we used displays only a very weak red birefringence. The distribution of connective tissue deposition seemed to coincide with the zones of organization of the astrocytosis into the gel. Although the collagen could form dense layers locally, we never observed the formation of a collagenous scar that was continuous all around the implantation site. Furthermore, collagen was also deposited inside $\mathrm{p}$ (GMA)-MAA gels, constituting a birefringent matrix formed of thick strongly red to yellow collagen fibers and fine fibers displaying a green birefringence (Fig. 4D). Other extracellular materials were deposited in the hydrogel implants as seen in silver impregnated sections. Fine and weakly stained arrays of fibrinogranular material were observed at the interface and also in p(GMA)-MAA gels. In this case, the network of this material could depict the microgeometric patterns of the hydrogel (Fig. 4C). Because of its argyrophilic properties, this material may correspond to reticulin fibers or basement membranes but neurites of small caliber should not be excluded. Elastic fibers were also seen as their amorphous components stained with PTAH, but these were mostly restricted to the interface.

\section{The axonal reaction}

The neuronal cell bodies of the cortex adjacent to the site of implantation showed either an intact morphology, chromatolysis or different degrees of atrophy (Fig. 6A). A constant observation, independent of the ionic structure of the hydrogels, was the presence of nerve fiber sprouts in the cortex surrounding the implant. These sprouts, which typically showed wavy patterns and swollen tips, were often arranged in fascicules terminating on the brain-implant interface (Fig. 6C, Table 1). Regenerating nerve fibers, either packed or individual, were also seen in the cortex adjacent to the implantation site (Fig. 6D). Three kinds of figures could generally be observed: regenerating cortical nerve fibers running approximately perpendicularly to one edge of the implant, or projecting into the implantation site, either following a direct trajectory or after deflection of their initial orientation. Despite the difficulty of establishing the relationship between the entering nerve fibers and the hydrogels because of the differing refractive index of the structure of the gel, it was nevertheless clear that in some cases individual axons did enter the hydrogel (Fig. 5C). This was mostly observed with $\mathrm{p}(\mathrm{GMA})$-MAA gels (Table 1). For the same reason, it was difficult to trace the course and to evaluate the exact extent of the nerve fibers penetrating the matrix.

\section{DISCUSSION}

Previously, we implanted neutral p(GMA) hydrogels into the brain in order to provide a support that could facilitate and direct the 

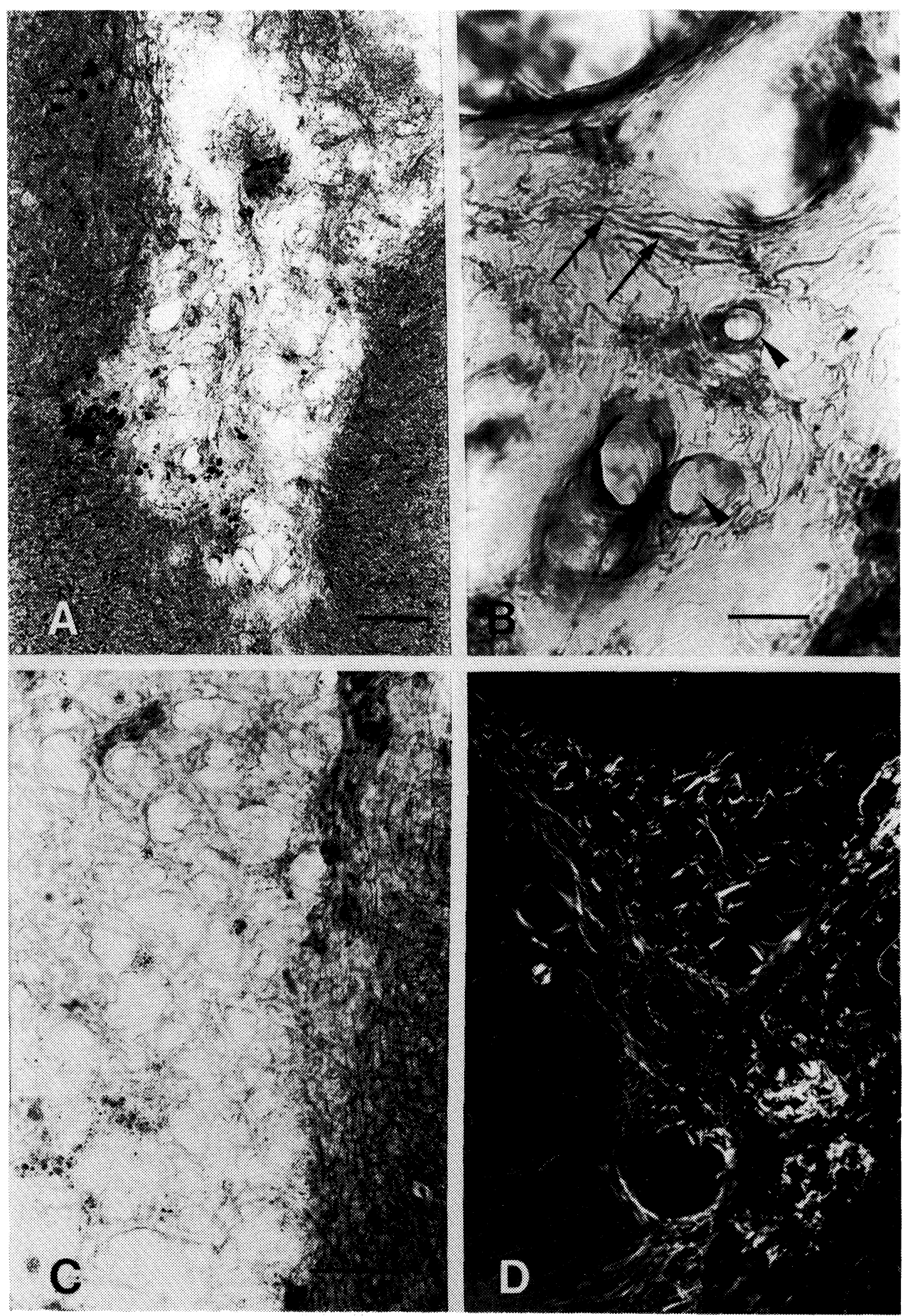

Fig. 4: $\quad p(G M A)-M A A$ hydrogel at 18 weeks post-implantation. $A$ and $B$ are PTAH stained sections showing the astrocytic reaction. In $A$ the astrocytic processes penetrated the gel massively, forming an astroglial matrix. Note also the weak glial reaction around the implant. B is a high magnification of a region of the gel interfacing with the neural tissue. Astrocytic processes (arrows) and blood vessels (arrowheads) are seen to penetrate the gel. C (Bodian) and D (picrosirius-polarization method) illustrate the deposition of argyrophilic material (C) and newly formed collagen (D) into the gel. Note that the staining pattern in Figures A, C and D reveals the cribriform matrix of the gel implant. Scale bars: A and C, $100 \mu \mathrm{m} ; \mathrm{B}, 20 \mu \mathrm{m}$ and $\mathrm{D}, 50 \mu \mathrm{m}$. 

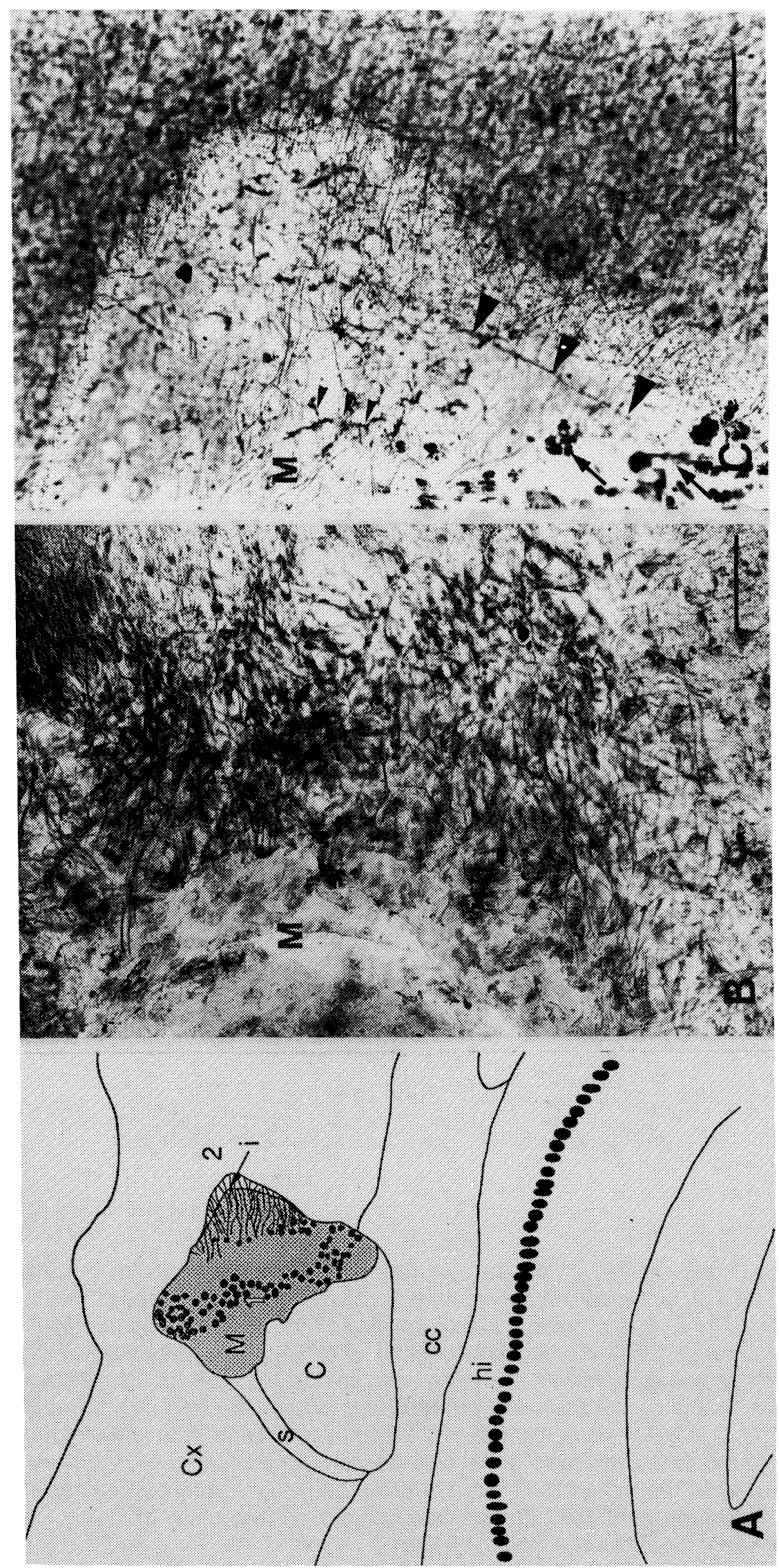

0

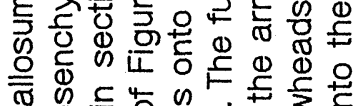
ठㅇำ

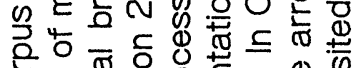

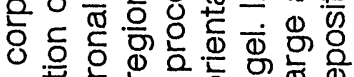

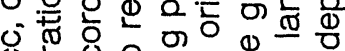
0.

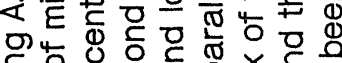

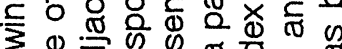

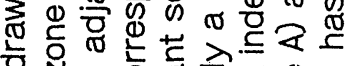

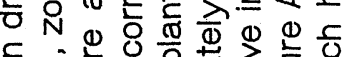

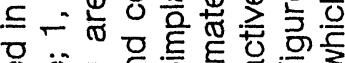

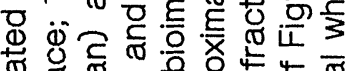

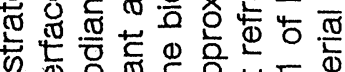

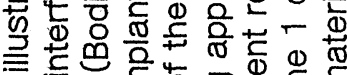
: $=0$. 范O

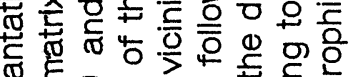

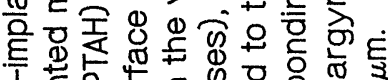

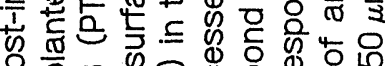

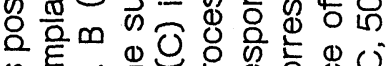
至

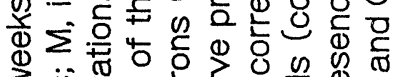
3 के

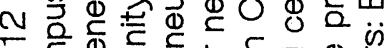

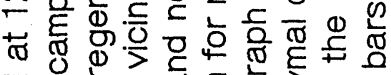
ब. 入ेट

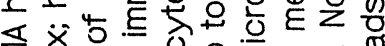

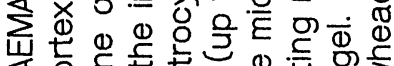

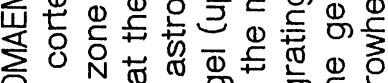

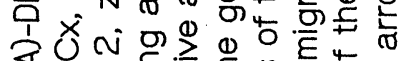

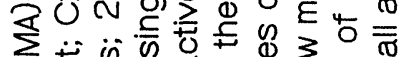

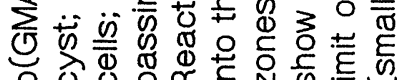
in 


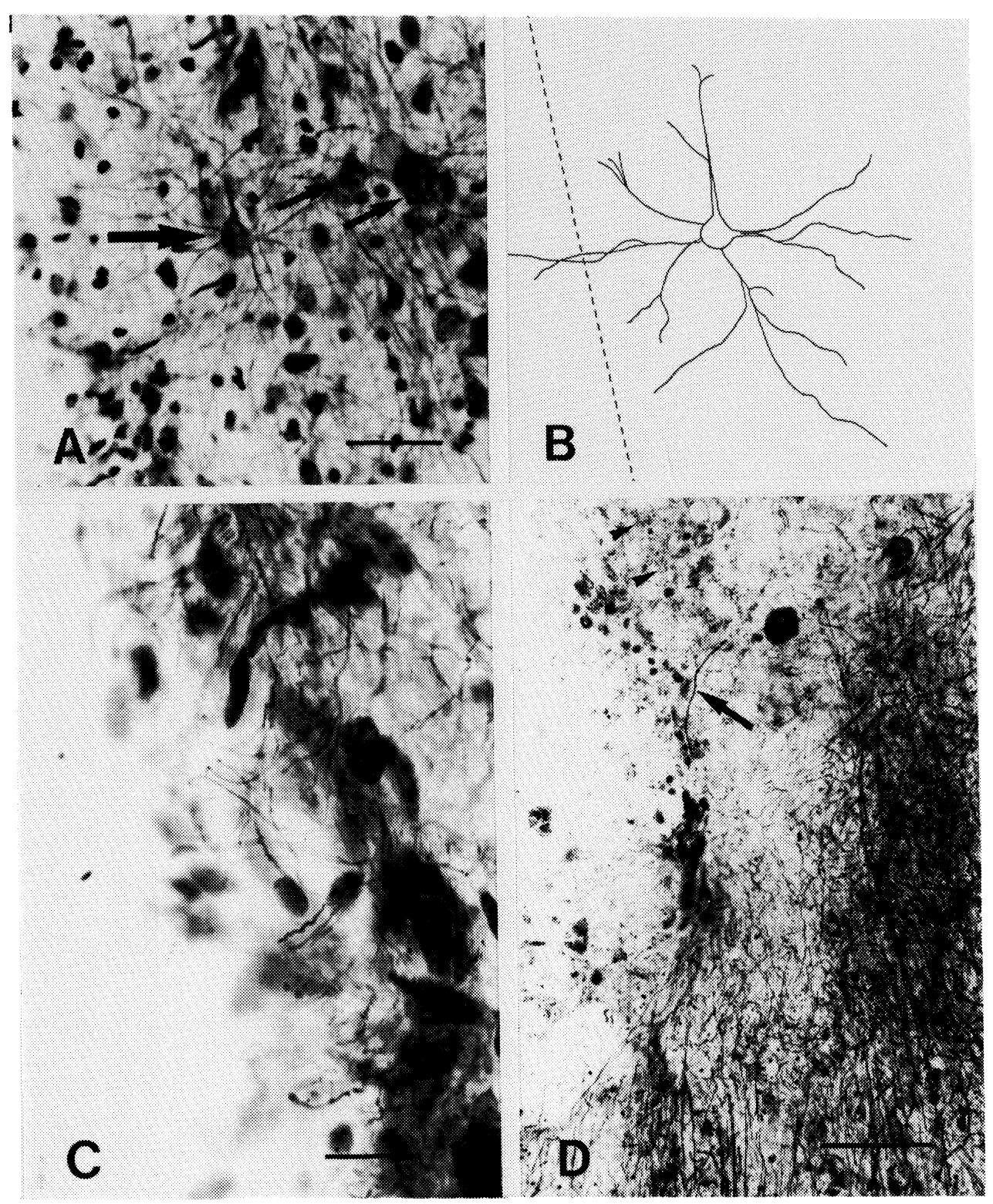

Fig. 6: Silver impregnated sections (Bodian) showing the neuronal response to the implantation of $p$ (GMA)-MAA gels after 18 weeks implantation. A, multipolar neurons with a normal morphology (arrows) located in the tissue adjacent to the implant. Figure $B$ is a camera lucida drawing of the neuron indicated by the large arrow in Figure A. This figure shows a ramified process projecting up to 50-60 $\mu \mathrm{m}$ away from the soma into the implantation site while other neurites (two of them show terminal ramifications) extend towards the margin of the implantation site. These processes could be traced by varying the field depth. The interface is indicated by the dashed line. $\mathrm{C}$ is a high magnification of the brain-implant interface showing nerve fiber sprouts often arranged in loose fascicules and projecting into the implantation site. D shows many regenerating nerve fibers that enter the implantation site. The arrow shows one process that has extended up to $150 \mu \mathrm{m}$ into the implantation site. The stained background corresponds to newly deposited argyrophilic material (arrowheads). Scale bars: A and D, $50 \mu \mathrm{m} ; \mathrm{C}, 10 \mu \mathrm{m}$. 
growth of cells and axons $/ 34 /$. In the present study, we incorporated polar chemical groups into the polymer skeleton. Our data show that these new $\mathrm{p}(\mathrm{GMA})$ hydrogels are well tolerated by the neural tissue and that they influence the gliosis which follows any injury of the brain parenchyma as well as an axonal response of the adjacent neural tissue. The influence of the ionic gels is demonstrated (1) by the mild astrocytosis along the hydrogel surface. This reaction is to be contrasted with that shown in our material beneath the implantation site at the level of the cut (a region which could be looked at as a lesion control); (2) by an increase and an organization of the growth of astrocyte processes onto and into the matrix. However, our findings show that the astrocytosis is more influenced by the hydrogels carrying carboxyl groups than those carrying amino groups. The deposition of extracellular material is also more pronounced with $\mathrm{p}(\mathrm{GMA})$ MAA hydrogels, but this could be related to different types of interactions between astrocytes and mesenchymal cells or extracellular molecules. The different behaviors of the glial reaction with respect to the type of gel can be interpreted in terms of these effects on the host tissue mediated through ionic currents and concentration gradients which take place in the matrices.

\section{Effect of ionic p(GMA) hydrogels on the astrocy- tosis}

The ionic currents are related to the charges of the hydrogels which acquire their charges by ionization and ion exchange in contact with ion containing media (collagen solution and interstitial fluid of the tissue). For instance, carboxyl and amino groups give the charged groups $\mathrm{COO}^{-}$ and $\mathrm{N}\left(\mathrm{CH}_{3}\right)_{2} \mathrm{H}^{+}$respectively (a scheme of the charged gels is given in Figure 7). However, 96\% of carboxyl groups are ionized while less than $1 \%$ of amino groups become charged at physiological $\mathrm{pH}$ (the level of charges of the gels is evaluated by measuring the $\mathrm{pH}$ of a diluted solution of comonomer and by calculating the constant of dissociation). The result is that $\mathrm{p}(\mathrm{GMA})-\mathrm{MAA}$ gels are highly polar materials with a high ionic conductivity while gels carrying basic groups are almost neutral. Ionic currents carried by highly mobile ions like $\mathrm{H}^{+}$may then have generated ion fluxes and favored the growth of cell processes toward the immobilized negative charges within the gel. Could ionic currents in the gels be related to the astrocytosis organization? Although ionic currents have not been measured, we believe that they may influence the elongation, the direction and the organization of cell processes. Indeed, ionic currents, generated by natural or applied electrical fields, have been shown to influence cellular events during regenerative processes in amphibians and in vertebrates $/ 6 /$ as well as during morphogenesis $/ 20 /$. The steady currents generated by the high level of charge of $\mathrm{p}(\mathrm{GMA})-\mathrm{MAA}$ gels may explain the massive invasion of astrocytic processes. Although contact guidance may also assist the growth of cell processes, voltage gradients are more potent cues /18/. Poly(GMA)-DMAEMA hydrogels behave more as neutral materials and the growth of glial processes could be modulated mainly through surface guidance mechanisms.
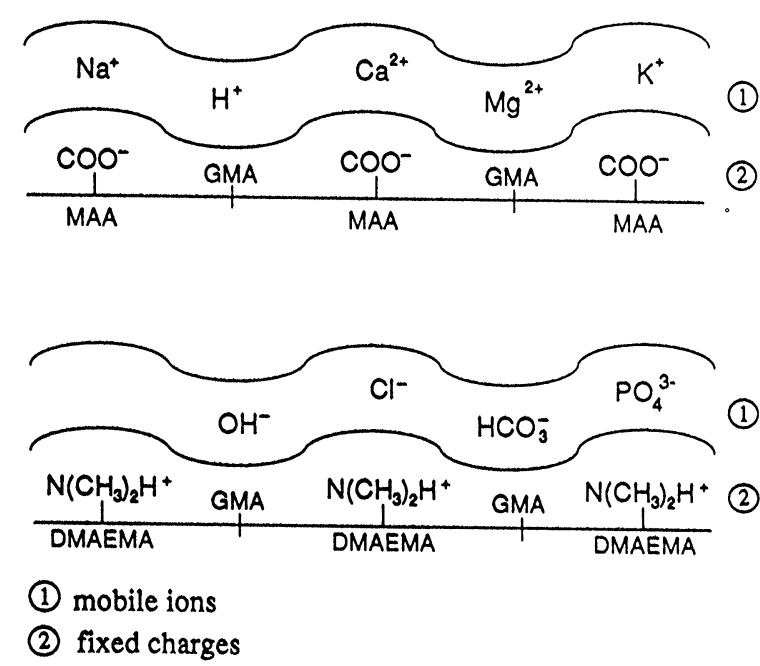

Fig. 7: Origin of the charges of $p(G M A)-M A A$ and $p(G M A)-D M A E M A$ hydrogels. The gels acquire their charges by ionization and/or ion adsorption in contact with polar media in order to satisfy the overall condition of electroneutrality. 
What are the mechanisms by which $\mathrm{p}$ (GMA)MAA-induced ionic currents can influence the astrocytosis? By analogy to galvanotaxis, where nerve fibers respond in vitro $/ 22 /$ and in vivo $126,5 /$ to an applied electric current, ionic currents may favor the accumulation of molecules involved in cell adhesion at the extemity of growing processes $/ 23 /$. Another possible and yet non-exclusive mechanism could be the buffering of injury cationic currents (mostly carried by $\mathrm{Na}^{+}$ and $\mathrm{Ca}^{++}$) /7/ which cause the depolymerization of the cytoskeleton of processes $/ 27 /$. Since the negative surface of $\mathrm{p}(\mathrm{GMA})-\mathrm{MAA}$ gels displays a strong affinity for positive ions, these gels could have buffered injury currents by attracting $\mathrm{Ca}^{++}$ (of all the cations available, calcium forms the most stable complex with acid functions), and the reduction of the $\mathrm{Ca}^{++}$currents could have reduced the extent of retrograde degeneration of processes.

Regardless of the ionic structure of the gels, the variable penetration of the hydrogels by the reactive host tissue is most likely related to the presence of collapsed pores at the surface of the gel or else to non-permeable microporous domains of the polymer as reported in our previous study $/ 34 /$ and by others $/ 29 /$. In addition to the ionic content of the synthetic gels, the collagen surface, the water content, the chemical inertness of the polymer skeleton and the permeability to gases and to low molecular weight solutes contribute to the biological properties of the hydrogels /24/.

\section{Regeneration of neurites}

Contrary to the astrocytic reaction where there were marked differences according to the types of gels, it seemed that the regenerating nerve fibers were more influenced by the topological organization of the astrocytosis rather than the type of gel (Fig. 4B and 4C). The implantation of charged hydrogels permits manipulation of two factors which are thought to limit the successful axonal regeneration in the CNS: formation of a connective/glial scar after injury of the neural tissue /14/ and lack of appropriate interactions of regenerating neurites with astrocytes $/ 17 /$. In our material, the $\mathrm{p}(\mathrm{GMA})$ MAA hydrogels, and to some extent the
p(GMA)-DMAEMA, modified the organization of the glial scar and this feature appears to become an important determinant for axonal regeneration. In particular, it is known that the persistence of a glial matrix promotes axonal regeneration after injury $/ 10 /$. Neuronoglial interactions are also thought to play a significant role in determining the outcome of neuroregenerative processes $/ 28 /$. In addition, the deposition of extracellular molecules and their organization onto the polymer surfaces should also be taken into account since extracellular matrix components can support and enhance neuritic outgrowth $/ 10 /$. Based on the argyrophilic properties of the material, these components may correspond, among other things, to reticulin fibers and/or basement membrane components /15/, secreted by reactive astrocytes $/ 3 /$. In our material, the extent to which astrocytic processes invaded $\mathrm{p}(\mathrm{GMA})-\mathrm{MAA}$ gels coincided with the distribution of secreted extracellular material.

\section{Comparison with a peripheral nerve graft}

Segments of peripheral nerve have been successfully implanted into the central nervous system to promote the growth of injured axons and it has been found to be a very effective procedure to stimulate axonal regeneration as a peripheral nerve graft may provide the adequate physical and trophic support for the growth of axons (e.g. Schwann cells and extracellular matrix molecules) with minimal gliotic reaction $12,31 /$. The use of synthetic polymeric matrices is an alternative procedure. The material implant itself is unable to support axonal growth but the aim here is to impose a spatial organization of the glial-connective scarring that could be conducive to directed axonal growth. Hydrogel implants induce a gliotic reaction which appears to be more intense than that occurring after the implantation of nerve segments $/ 4 /$, but the secondary organization of astrocytic processes in a glial-derived tissue matrix and the deposition of newly synthetized extracellular macromolecules are beneficial for axonal regeneration. In this regard, our biocompatible hydrogel, in contrast to a peripheral nerve implant which contains organized extracellular matrices and tubes of basal lamina $/ 8 /$, can be looked at as a "protomatrix" 
which acquires, after implantation, bioadhesive properties suitable to promote and support axonal growth while substrate pathways are orientated by the microgeometry of the matrix. However, as shown in the present study, this depends on the particular properties of the polymer surfaces, e.g., the presence of ionizable groups.

Although ionic matrices have been implanted in non-neural tissues $/ 1,30 /$, this is the first report, to our knowledge, of the implantation of polymer matrices carrying functional groups into brain tissue. This technology is a new addition to the presently available neurotransplantation methods that attempt to overcome the formation of scar tissue and consequently promote more effective axonal regeneration. Furthermore, the use of polar hydrogels may also allow manipulation of the cellular and the physico-chemical environment of the growing axons.

\section{ACKNOWLEDGEMENTS}

This research was supported by the Medical Research Council of Canada (MRC, grant MT 6797 to R.M.) and the Fonds de la recherche en santé du Québec (FRSQ).

The authors express their sincere gratitude to Mrs. Louise Bertrand and Nicole Massicotte for their technical assistance.

\section{REFERENCES}

1. Barvic M, Vacik J, Lim D, Zavadil M. Tolerance of modified poly(glycol methacrylates) by the organism. J Biomed Mater Res 1971; 5: 225-238.

2. Benfey M, Aguayo AJ. Extensive elongation of axons from rat brain into peripheral nerve grafts. Nature 1982; 296: 150-152.

3. Bernstein JJ, Getz R, Jefferson M, Kelemen $M$. Astrocytes secrete basal lamina after hemisection of rat spinal cord. Brain Res 1985; 327: 135-141.

4. Berry M, Hall S, Follows R, Rees L, Gregson N, Sievers J. Response of axons and glia at the site of anastomosis between the optic nerve and cellular or acellular sciatic nerve grafts. J Neurocytol 1988; 17: 727-744.

5. Borgens RB, Blight AR, Murphy DJ, Stewart L. Transected dorsal column axons within the guinea pig spinal cord regenerate in the presence of an applied electrical field. J Comp Neurol 1986; 250: 168-180.
6. Borgens $\mathrm{RB}$. What is the role of naturally produced electric current in vertebrate regeneration and healing? Int Rev Cytol 1982; 76: 245-298.

7. Borgens RB, Jaffe LF, Cohen MJ. Large and persistent electrical currents enter the transected lamprey spinal cord. Proc Natl Acad Sci USA 1980; 77: 1209-1213.

8. Bunge MB, Bunge RP, Kleitman N, Dean AC. Role of peripheral nerve extracellular matrix in Schwann cell function and in neurite regeneration. Dev Neurosci 1989; 11: 348-360.

9. Civerchia-Perez L, Faris B, La Pointe G, Beldras J, Leibowitz $H$, Franzblau C. Use of collagenhydroxymethacrylate hydrogels for cell growth. Proc Natl Acad Sci USA 1980; 77: 2064-2068.

10. Carbonetto S, Douville P, Harvey W, Turner DC. Laminin, fibronectin, collagen and their receptors in nerve fiber growth. In: Reier JP, Bunge RP, Seil FJ, eds, Current Issues in Neural Regeneration Research. New York: A.R. Liss, 1988; pp. 147-158.

11. Collins GH, West NR. Glial activity during axonal regrowth following cryogenic injury of rat spinal cord. Brain Res Bull 1989; 22: 71-79.

12. Junqueria LC, Cossermelli W, Brentani RR. Differential staining of collagen type I, II and III by sirius red and polarization microscopy. Arch Histol Jpn 1978; 41: 267-274.

13. Junqueria LC, Bignolas G, Brentani RR. Picrosirius staining plus polarization microscopy, a specific method for collagen detection in tissue section. Histochem J 1979; 11: 447-455.

14. Kiernan JA. Hypotheses concerned with axonal regeneration in the mammalian nervous system. Biol Rev 1979; 54: 153-197.

15. Leblond CP, Laurie GW. Morphological features of connective tissues. Rheumatology 1986; 10: 1-28.

16. Madison R, Sidman RL, Nyilas E, Chiu T-H, Greatorex D. Nontoxic nerve guide tubes support neovascular growth in transected rat optic nerve. Exp Neurol 1984; 86: 448-461.

17. Manthorpe M, Rudge JS, Varon S. Astroglia cell contributions to neuronal survival and neuritic growth. In: Federoff S, Vernadakis A, eds, Astrocytes: Biochemistry, Physiology and Pharmacology, Vol. 2. New York: Academic Press, 19 ; pp.

18. McCaig $C D$. Electric fields, contact guidance and the direction of nerve growth. J Embryol Exp Morph 1986; 94: 245-255.

19. Melville S, Sherburn TE, Coggeshall RE. Preservation of sensory cell by placing stumps of transected nerve in an impermeable tube. Exp Neurol 1989; 105: 311-315.

20. Nuccitelli R. Ionic currents in morphogenesis. Experientia 1988; 4: 657-666.

21. Nyilas E, Chiu T-H, Sidman RL, Henry EW, Brushart TM, Dikkes P, Madison R., Peripheral nerve repair with bioresorbable prosthesis. Trans Am Soc Artif 
Intern Organs 1983; 29: 307-312.

22. Patel N, Poo M-M. Orientation of neurite growth by extracellular electrical fields. J Neurosci 1982; 2: 483496.

23. Poo M-M. In situ electrophoresis of membrane components. Ann Rev Biophys Bioeng 1981; 10: 245276.

24. Ratner RD, Hoffman AS. Synthetic hydrogels for biomedical applications. In Andrade JD, ed, Hydrogels for Medical and Related Applications. Washington, DC: ACS Symposium Series 31, 1976; pp 1-35.

25. Refojo MF. Glyceryl methacrylate hydrogels. J Appl Polymer Sci 1965; 9: 3161-3170.

26. Roederer E, Goldberg NH, Cohen MJ. Modification of retrograde degeneration in transected spinal axons of the lamprey by applied DC current. Neurosci 1983; 3: $153-160$.

27. Schlaepfer WW, Hasler MB. Characterization of the calcium-induced disruption of neurofilaments in rat peripheral nerve. Brain Res 1979; 168: 299-309.

28. Silver J, Lorenz SE, Wahlsten D, Coughlin J. Axonal guidance during development of the great cerebral commissure: descriptive and experimental studies, in vivo, on the role of preformed glial pathways. J Comp Neurol 1982; 210: 10-29.

29. Sprincl L, Kopocek J, Lim D. Effect of porosity of heterogeneous poly(glycol monomethacrylate) gels on the healing-in of test implants. J Biomed Mater Res 1971; 4: 447-458.

30. Sprincl L, Vacik J, Kopocek J. Biological tolerance of ionogenic hydrophilic gels. J Biomed Mater Res 1973; 7: 132-136.

31. Weinberg EL, Raine CS. Reinnervation of peripheral nerve segments implanted into the rat central nervous system. Brain Res 1980; 198: 1-11.

32. Wichterle O, Lim D. Hydrophilic gels for biological use. Nature 1960; 185: 117-118.

33. Woerly S, Marchand R. Intracerebral implantation of ionogenic matrices. Soc Neurosci Abstr 1989; 15: 1370.

34. Woerly $S$, Marchand $R$, Lavallée $C$. Intracerebral implantation of synthetic plymer/biopolymer matrix: a new perspective for brain repair. Biomaterials 1990; 11: 97-107. 

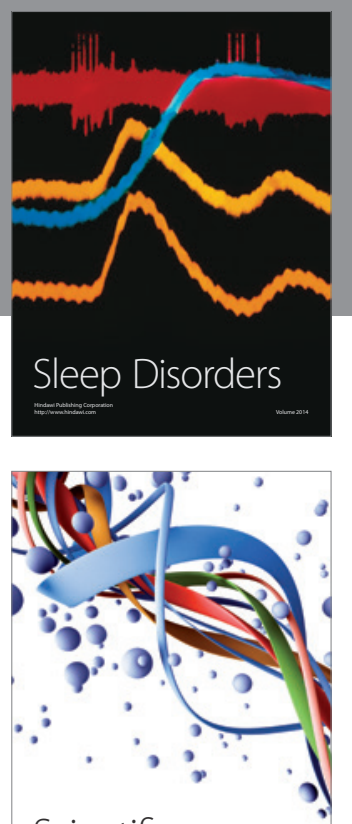

Scientifica
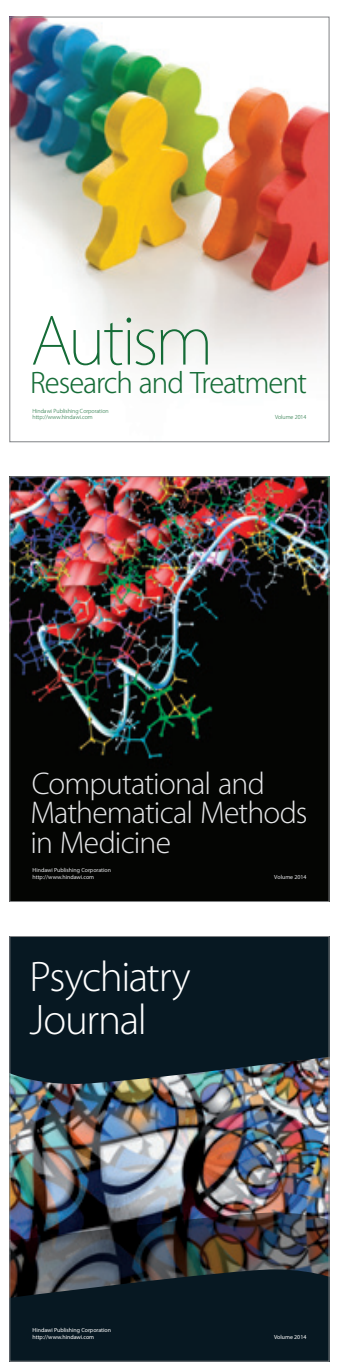
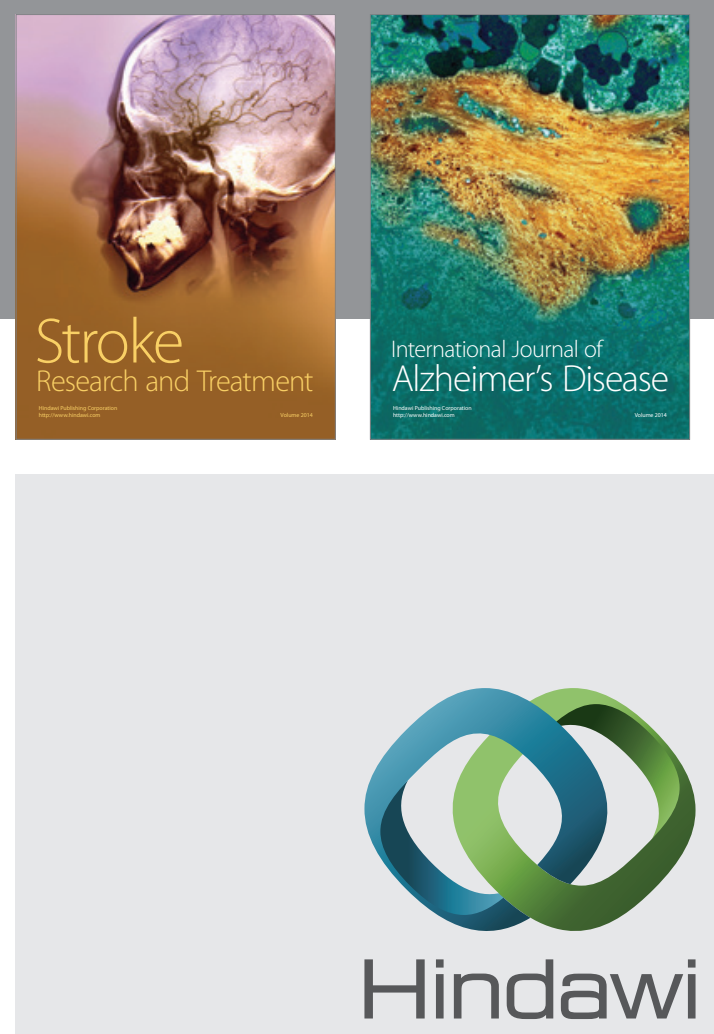

Submit your manuscripts at

http://www.hindawi.com
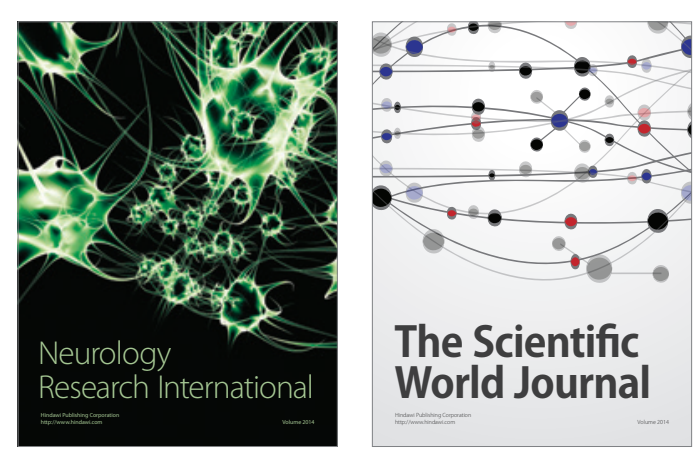

The Scientific World Journal

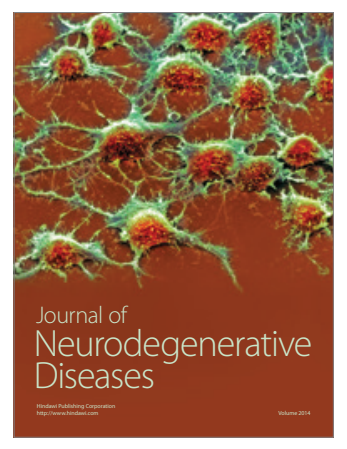

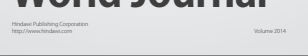

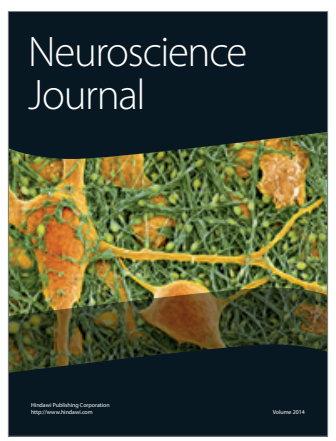

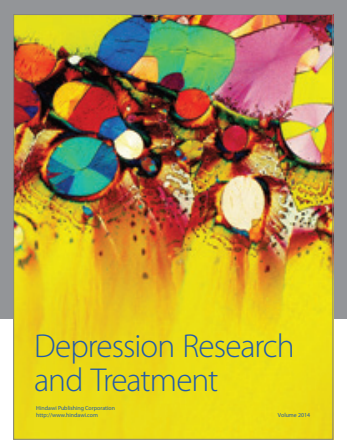
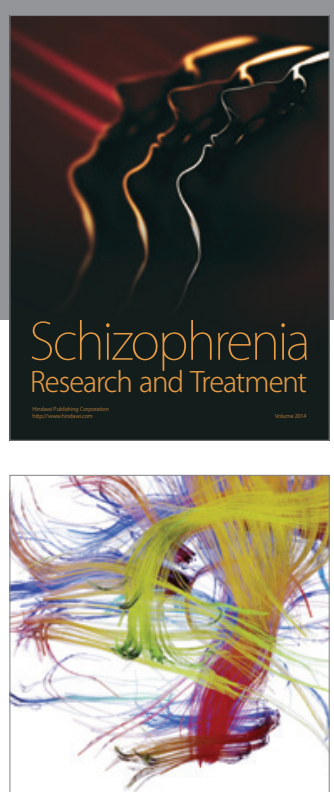

Brain Science

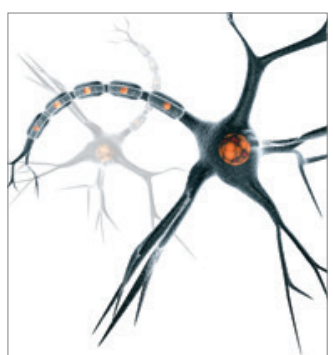

Neural Plasticity
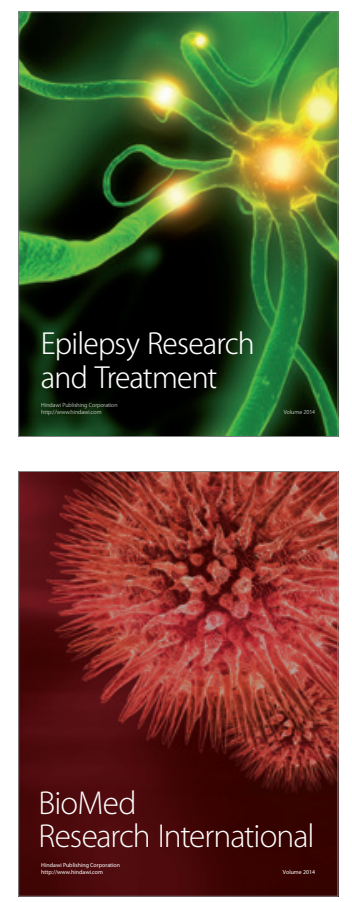

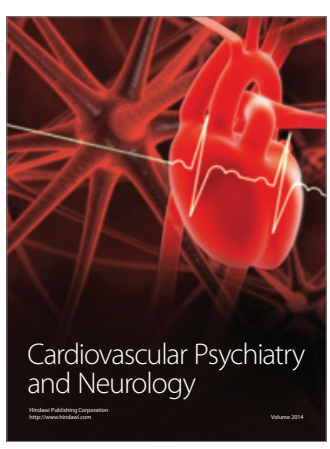

Parkinson's

Disease
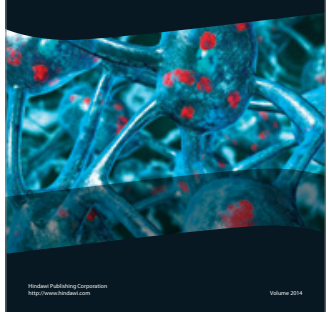